Physics

Physics Research Publications

\title{
A new search for primordial black hole evaporations using the Whipple gamma-ray telescope
}

E. T. Linton, R. W. Atkins, H. M. Badran, G. Blaylock, P. J. Boyle, J. H. Buckley, K. L. Byrum, D. A. Carter-Lewis, O. Celik, Y. C. K. Chow, P. Cogan, M. K. Daniel, C. Dowdall, A. D. Falcone, D. J. Fegan, S. J. Fegan, J. P. Finley, P. Fortin, K. J. Guiterrez, J. Hall, D. Hanna, J. Holder, D. Horan, S. B. Hughes, T. B. Humensky, I. Jung, G. E. Kenny, M. Kertzman, D. B. Kieda, J. Kildea, J. Knapp, H. Krawczynski, M. J. Lang, S. LeBohec, G. Maier, P. Moriarty, R. A. Ong, J. S. Perkins, F. Pizlo, M. Pohl, J. Quinn, K. Ragan, P. F. Rebillot, P. T. Reynolds, G. H. Sembroski, D. Steele, S. P. Swordy, L. Valcarcel, S. P. Wakely, T. C. Weekes, and R. J. White

This paper is posted at Purdue e-Pubs.

http://docs.lib.purdue.edu/physics_articles/317 


\section{A new search for primordial black hole evaporations using the Whipple gamma-ray telescope}

E T Linton ${ }^{1}$, R W Atkins ${ }^{2}$, H M Badran ${ }^{3}$, G Blaylock ${ }^{4}$, P J Boyle ${ }^{1}$, J H Buckley ${ }^{5}, \mathrm{~K}$ L Byrum ${ }^{6}$,

D A Carter-Lewis ${ }^{7}, \mathrm{O}$ Celik $^{8}$, Y C K Chow ${ }^{8}$, P Cogan 9 , M K Daniel ${ }^{9}$, C Dowdall ${ }^{9}$, A D Falcone ${ }^{10}$, D J Fegan ${ }^{9}$, S J Fegan ${ }^{8}$, J P Finley ${ }^{11}$, P Fortin ${ }^{12}$, K J Guiterrez ${ }^{5}$, J Hall ${ }^{2}$, D Hanna ${ }^{13}$, J Holder ${ }^{14}$, D Horan ${ }^{15}$, S B Hughes ${ }^{5}$, T B Humensky ${ }^{1}$, I Jung ${ }^{5}$, G E Kenny ${ }^{16}$, M Kertzman ${ }^{17}$, D B Kieda ${ }^{2}$, J Kildea ${ }^{13}$, J Knapp ${ }^{14}$, H Krawczynski ${ }^{5}$, M J Lang ${ }^{16}$, S LeBohec ${ }^{2}$, G Maier ${ }^{14}$, P Moriarty ${ }^{18}$, R A Ong ${ }^{8}$, J S Perkins ${ }^{5}$, F Pizlo ${ }^{11}$, M Pohl $^{7}$, J Quinn ${ }^{9}$, K Ragan ${ }^{13}$, P F Rebillot ${ }^{5}$, P T Reynolds ${ }^{19}$, G H Sembroski ${ }^{11}$, D Steele ${ }^{20}$, S P Swordy ${ }^{1}$, L Valcarcel ${ }^{13}$, S P Wakely ${ }^{1}$, T C Weekes ${ }^{15}$ and R J White ${ }^{14}$

${ }^{1}$ Enrico Fermi Institute, University of Chicago, Chicago, IL 60637, USA

2 Physics Department, University of Utah, Salt Lake City, UT 84112, USA

${ }^{3}$ Department of Physics, Faculty of Science, Tanta University, Tanta 31527,

Egypt

${ }^{4}$ Department of Physics, University of Massachusetts, Amherst, MA 01003-4525, USA

${ }^{5}$ Department of Physics, Washington University, St Louis, MO 63130, USA

6 Argonne National Laboratory, 9700 S. Cass Avenue, Argonne, IL 60439, USA

7 Department of Physics and Astronomy, Iowa State University, Ames, IA 50011, USA

${ }^{8}$ Department of Physics and Astronomy, University of California, Los Angeles, CA 90095, USA

${ }^{9}$ School of Physics, University College Dublin, Belfield, Dublin 4,

Republic of Ireland

10 Department of Astronomy and Astrophysics, Penn State University,

University Park, PA 16802, USA

11 Department of Physics, Purdue University, West Lafayette, IN 47907, USA

12 Department of Physics and Astronomy, Barnard College, Columbia University, NY 10027, USA

13 Physics Department, McGill University, Montreal, QC H3A 2T8, Canada 
${ }^{14}$ School of Physics and Astronomy, University of Leeds, Leeds, LS2 9JT, UK

${ }^{15}$ Fred Lawrence Whipple Observatory, Harvard-Smithsonian Center for

Astrophysics, Amado, AZ 85645, USA

${ }^{16}$ Physics Department, National University of Ireland, Galway,

Republic of Ireland

17 Department of Physics and Astronomy, DePauw University, Greencastle, IN 46135-0037, USA

18 Department of Physical and Life Sciences, Galway-Mayo Institute of

Technology, Dublin Road, Galway, Republic of Ireland

${ }^{19}$ Department of Applied Physics and Instrumentation, Cork Institute of

Technology, Bishopstown, Cork, Republic of Ireland

20 Astronomy Department, Adler Planetarium and Astronomy Museum,

Chicago, IL 60605, USA

E-mail: etl@uchicago.edu

Received 21 December 2005

Accepted 24 December 2005

Published 16 January 2006

Online at stacks.iop.org $/ \mathrm{JCAP} / 2006 / \mathrm{i}=01 / \mathrm{a}=013$

doi:10.1088/1475-7516/2006/01/013

Abstract. Stephen Hawking's prediction that black holes should radiate like black bodies has several important consequences, including the possibility of the detection of small $\left(\sim 10^{15} \mathrm{~g}\right)$ black holes created in the very early universe. The detection of such primordial black holes (PBHs) would be an important discovery, not only confirming Hawking's theory, but also providing valuable insights into the history of the early universe. A search through 5.5 years of archival data from the Whipple Atmospheric Cerenkov Telescope is made for TeV gamma-ray bursts on 1,3 , and $5 \mathrm{~s}$ timescales. On the basis of a null result from this direct search for PBH evaporations, an upper limit of $1.08 \times 10^{6} \mathrm{pc}^{-3} \mathrm{yr}^{-1}(99 \% \mathrm{CL})$ is set on the $\mathrm{PBH}$ evaporation rate in the local region of the galaxy, assuming the Standard Model of particle physics. This is more than a factor of two better than the previous limit at this energy range and includes longer timescales than have previously been explored. Comparison of this result with previous limits on the fraction of the critical density comprised by PBHs, $\Omega_{\mathrm{pbh}}$, depends strongly on assumptions made about $\mathrm{PBH}$ clustering; in models predicting strong $\mathrm{PBH}$ clustering, the limit in this work could be as many as ten orders of magnitude more stringently than those set by diffuse $\mathrm{MeV}$ gamma-ray observations.

Keywords: gamma ray bursts, black holes 


\section{Contents}

1. Introduction 3

2. Data collection 4

2.1. The Whipple $10 \mathrm{~m}$ telescope . . . . . . . . . . . . . . . . 4

2.2. The data set . . . . . . . . . . . . . . . . . . . . . 5

2.3. Gamma-ray selection . . . . . . . . . . . . . . . . 5 5

3. Burst-search strategy $\quad 6$

3.1. Selecting bursts . . . . . . . . . . . . . . . . . . 6

3.2. Determining background . . . . . . . . . . . . . . . . 7

3.3. Theoretical signal . . . . . . . . . . . . . . . . . . . 7

3.4. Theoretical model for the final stages of $\mathrm{PBH}$ evaporation . . . . . . . . . 8

4. Results 8

5. Conclusions 9

Acknowledgments 11

References 11

\section{Introduction}

Stephen Hawking's 1974 prediction that black holes should radiate as black bodies with a temperature $T_{\mathrm{BH}}=\left(\hbar c^{3} / 8 \pi G\right)(1 / M)$ was remarkable for several reasons [1]. One was that it was the first example of a phenomenon to be described using both quantum mechanics and general relativity. A second was that it overturned the notion that black holes could only absorb and never emit particles.

Although theoretically interesting, it at first seemed unlikely that this radiation would ever actually be observed. This is because the smallest black holes that can form from stellar collapse would have a mass on the order of the sun's, resulting in a temperature of $\sim 10^{-7} \mathrm{~K}$. However, density perturbations present in the early universe could have collapsed into black holes of much smaller masses and, consequently, much higher temperatures. Such black holes are referred to as primordial black holes (PBHs).

$\mathrm{PBH}$ lose mass at a rate of

$$
\frac{\mathrm{d} M}{\mathrm{~d} t}=-\frac{\alpha(M)}{M^{2}}
$$

due to Hawking radiation [2]. The increase in the evaporation rate as the mass decreases leads to a runaway process that, depending on the physics governing the final stages of evaporation, could result in a violent explosion. The factor $\alpha(M)$ depends on the degrees of freedom available to the radiated particles at a given temperature. Consequently, the exact fate of a $\mathrm{PBH}$ as its mass drops toward zero is an open question since it is governed by particle physics at energies above those accessible by current accelerators. The limits presented in this work assume no additional degrees of freedom beyond the Standard Model of particle physics. 
If PBHs are detected, the energy range at which they are seen would help determine the number of particle degrees of freedom at these higher energies. Their detection would also provide valuable cosmological information on the early universe, including information on density perturbations and phase transitions [3].

The best chance for direct detection of the final explosion of a $\mathrm{PBH}$ lies in viewing the resulting burst of gamma rays, since they are both electrically neutral and have a useful interaction cross-section. Another signature of $\mathrm{PBH}$ evaporations is antiproton emissions [4]. These have been used to place tight limits on PBH densities, but they cannot be used to view individual bursts directly due to deflections by magnetic fields. Neutrinos are also expected to be emitted by $\mathrm{PBHs}$, but the low interaction cross-section and larger background make them less useful in PBH searches. The idea of using gamma rays to search for PBH evaporations is not new, and in fact, the Whipple $10 \mathrm{~m}$ telescope has been used in such searches for many years [5]. Improvements in telescope hardware, analysis techniques, and our theoretical understanding of PBHs have all caused the resulting limits to change significantly over time.

The theoretical uncertainties at higher energies mentioned above, as well as other uncertainties in the emission mechanism, could significantly affect the detectability of PBHs. For example, increasing the number of degrees of freedom available at higher PBH temperatures, as in the Hagedorn model [6], will increase the evaporation rate and cause its output to peak at a lower energy. This model is one of the motivations behind the Short GAmma Ray Front Air Cerenkov Experiment (SGARFACE), which is run at the Whipple $10 \mathrm{~m}$ telescope in parallel with its normal observations and is sensitive to microsecond-timescale bursts down to an energy of $100 \mathrm{MeV}$ [7]. Another possibility is that a photosphere might form around a $\mathrm{PBH}$, thermalizing the radiation [8]. In such a scenario any $\mathrm{TeV}$ signature would be drastically reduced.

The remainder of this paper first outlines the method used for collecting the data using the Whipple $10 \mathrm{~m}$ telescope (section 2) and then discusses the specific burst-search strategy (section 3). The results are then presented, and in the absence of a signal an upper limit is set (section 4). Finally, this upper limit is compared with previous results (section 5).

\section{Data collection}

\subsection{The Whipple $10 \mathrm{~m}$ telescope}

The Whipple $10 \mathrm{~m}$ telescope has been used for gamma-ray astronomy since 1968 . The telescope consists of a $10 \mathrm{~m}$ diameter Davies-Cotton reflector with a fast photomultiplier tube (PMT) camera in the focal plane and is located at an altitude of $2300 \mathrm{~m}$ above sea level in southern Arizona. It detects gamma rays by imaging the Cerenkov light from the air showers they produce upon entering the Earth's atmosphere. More details about this technique can be found elsewhere [9]. The sensitive energy range of the instrument spans from approximately $200 \mathrm{GeV}$ to greater than $20 \mathrm{TeV}$.

Starting in 1997 the Whipple telescope used a camera with 331 PMTs for a field of view (FOV) of $4.8^{\circ}$. The camera was triggered if any three of the 331 pixels each produced a signal $\geq 65$ photoelectrons with an effective resolving time of 8 ns. In 1999 a more sophisticated triggering system, known as the pattern selection trigger, was installed [10]. 
This new trigger system reduced the number of accidentals, allowing the telescope to be run at a lower threshold while keeping the trigger rate at a manageable level of around $40 \mathrm{~Hz}$. Since the fall of 2000, the Whipple telescope has been equipped with the GRANITE-III high resolution camera. This camera consists of 379 PMTs each with an angular size of $0.12^{\circ}$, giving a total FOV of about $2.3^{\circ}$.

\subsection{The data set}

This work covers data taken from January of 1998 through July of 2003 and is divided into three periods. The first period starts in January 1998 and continues through the spring of 1999, during which time the 331-PMT camera was used. The 379-PMT camera was installed in the summer before the 1999-2000 observing season, which makes up the second period. The upgrade was not fully complete until the fall of 2000 , and so the third period begins there and continues through the spring of 2003. Over these five and a half years $2191 \mathrm{~h}$ of data in good weather were taken.

Each of these three periods is further subdivided into three zenith angle (ZA) bins: $\mathrm{ZA} \leq 20^{\circ}, 20^{\circ}<\mathrm{ZA} \leq 40^{\circ}$, and $40^{\circ}<\mathrm{ZA}$. This is done because the effective collection area of the telescope is dependent on the ZA, and it is desirable to combine data sets with similar gamma-ray rates so as not to mask any potential signal in the lower rate data.

One factor that aids in this kind of search is that it can be carried out without interfering with the telescope's normal observing schedule. Even data taken with a known source within the FOV can still be used since source rates are typically on the order of a few counts per minute and so would have little effect on a search for secondscale bursts. In addition, the method used for estimating the background (section 3.2) automatically eliminates the effects of steady sources. If a very strong source (such as an active galactic nucleus in a flaring state) were producing second-scale bursts, such a signal may be detected. However, such a result is not observed, and would have been interesting in its own right anyway.

\subsection{Gamma-ray selection}

The primary background for ACTs is showers initiated by cosmic rays. These showers occur on the same timescales as gamma-ray initiated showers, but they tend to be less compact spatially. Based on this difference, a technique that involves calculating the zeroth, first, and second moments of each image has been successfully used to discriminate between hadron and gamma-ray induced showers [11,12]. For point sources it is capable of rejecting greater than $99 \%$ of the background while retaining greater than $50 \%$ of the gamma-ray signal.

The standard analysis method has been optimized for when sources lie directly along the telescope's pointing axis. For this search, however, it is useful to be able to use the entire FOV of the camera since the potential source locations are not known before hand. Thus the method of Lessard [13] for determining the arrival directions for all detected events, regardless of their location in the image plane, is used.

Although sources not at the centre of the FOV can still be detected, the further off-axis the arrival direction of a primary gamma ray is, the less likely it is that the shower image will fall within the camera's FOV. Consequently, the collection area of the telescope decreases with increasing offset angle, $\theta$. In section 3.3 we make the simplifying 

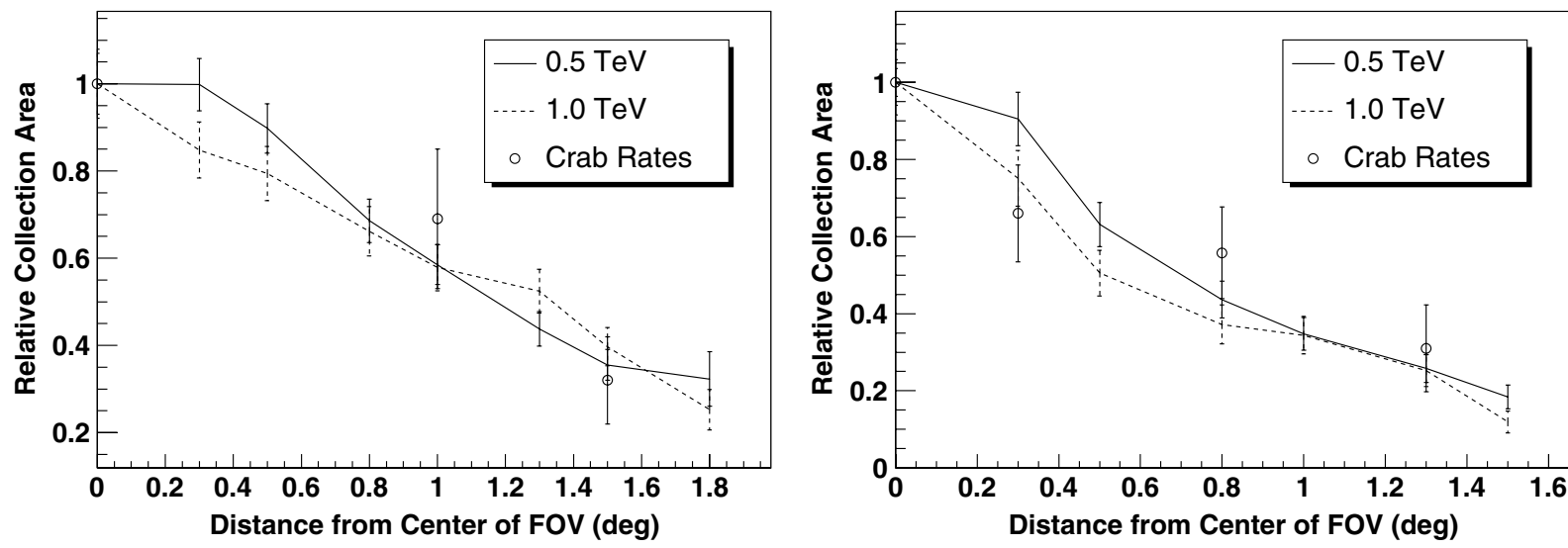

Figure 1. Simulated off-axis response for the Whipple camera compared with rates for the Crab Nebula taken with the telescope offset from the true source position. The plot on the left is for the 331-PMT camera, with Crab rates from reference [13]. The plot on the right is for the most recent configuration of the 379-PMT camera.

assumption that the energy dependence and the angular offset dependence of the collection area can be factored such that the effective area $A(E, \theta)=A_{\circ}(E) a(\theta)$, with $a(0)=1$. The KASCADE simulation package [14] is used to determine $A_{\circ}(E)$ and $a(\theta)$. The off-axis sensitivity is verified using data taken from a steady point source, the Crab Nebula, with the telescope deliberately offset from the true source position (figure 1). It can be seen that the separation of the collection area into $E$ and $\theta$ dependent parts holds fairly well for the energies tested.

\section{Burst-search strategy}

\subsection{Selecting bursts}

Calculations assuming the Standard Model of particle physics indicate that detectable fluxes of $\mathrm{TeV}$ gamma rays are produced during the last few seconds of a PBH's life [2]. A previous work made a search for $\mathrm{TeV}$ gamma-ray bursts within a time window, $\Delta t$, of $1 \mathrm{~s}$ using data taken by the Whipple telescope from 1988 to 1992 [15]. Due to difficulties in predicting the background rate, selecting the optimal time window for the search a priori is not straightforward. Consequently, in this current work bursts of 1,3 , and 5 s durations are sought.

In addition to all the events of a burst falling within a given time window, it is also required that their arrival directions overlap to within the angular resolution of the camera $\left(\sigma \approx 0.13^{\circ}\right)$. This is equivalent to saying that some circle with a radius equal to the angular resolution, $\sigma$, contains all the event arrival directions. With the time constraint added, a burst can then be defined as a group of events falling within a space-time cylinder of height $\Delta t$ and radius $\sigma$. The size of a burst is defined as the number of events within that burst.

There are a number of issues involved when deciding how to count bursts, such as whether to count sub-bursts of larger bursts and how to count bursts that overlap in time 
or space. In this study we select all the potential gamma-ray events and then determine the size of the largest burst containing each of these events. The number of bursts, $N$, of each size, $b$, is then defined as the total number of events whose largest burst size is $b$, divided by $b$. This definition of $N(b)$ has the advantage that the total number of events $N_{\text {tot }}=\sum_{b=1}^{\infty} b N(b)$, which is the normalization one would expect for the number of bursts of size $b$. Note that due to the possibility of an event being contained in multiple bursts, in some cases $N(b)$ will not be an integer.

\subsection{Determining background}

Even in the absence of bursting sources, bursts are still observed due to random fluctuations in the rate of background events. However, an analytical calculation of what this background is expected to be is complicated by the fact that the camera is inhomogeneous. Different areas of the camera are more sensitive than others which leads to an artificial spatial clustering of events. In addition, clustering may be introduced by any nonuniformities in the sky brightness or by the presence of steady $\mathrm{TeV}$ gamma-ray sources. Consequently a method for estimating the background that automatically takes these factors into account has been developed [15].

First the time stamps of the data are scrambled, and then the gamma-ray-like events are selected. The same burst-search algorithm described above is then used; however, since the times are now essentially random, any real bursts caused by astrophysical phenomena are removed, leaving only the statistically generated bursts. This entire procedure is repeated ten times and the average results are taken as the background.

\subsection{Theoretical signal}

The expected number of gamma rays to be detected from a PBH at distance $r$ and angular offset $\theta$ over the final $\Delta t$ seconds of the PBH's life is given by,

$$
N_{D}(r, \theta, \Delta t)=\frac{a(\theta)}{4 \pi r^{2}} \int_{0}^{\Delta t} \mathrm{~d} t \int_{0}^{\infty} \mathrm{d} E \frac{\mathrm{d}^{2} N}{\mathrm{~d} E \mathrm{~d} t}(E, t) A_{\circ}(E) \equiv \frac{a(\theta)}{4 \pi r^{2}} I
$$

where $\mathrm{d}^{2} N / \mathrm{d} E \mathrm{~d} t$ is the emission rate for gamma rays of energy $E$ from a $\mathrm{PBH}$ at time $t$ before total evaporation. The factors $A_{\circ}(E)$ and $a(\theta)$ refer to the collection area of the telescope and are defined in section 2.3. All the PBH physics has been moved into the factor $I$ in order to simplify the insertion of different theoretical models.

The probability of observing a burst of $b$ events within a time window $\Delta t$ from a $\mathrm{PBH}$ at coordinates $r$ and $\theta$ depends only on $N_{D}(r, \theta, \Delta t)$ and $b$. Calling this probability $P\left(b, N_{D}(r, \theta, \Delta t)\right)$ and integrating over space, we find the total number of bursts of size $b$ :

$$
n_{s}(b, \Delta t)=\rho_{\mathrm{pbh}} \tau \int \mathrm{d} \Omega \int_{0}^{\infty} \mathrm{d} r r^{2} P\left(b, N_{D}(r, \theta, \Delta t)\right),
$$

where $\tau$ is the total observation time and $\rho_{\mathrm{pbh}}$ is the number of $\mathrm{PBH}$ evaporations per unit volume per unit time. In the previous search [15], $P\left(b, N_{D}\right)$ is taken to be $\Theta\left(N_{D}(r, \theta, \Delta t)-b\right)$, where $\Theta(x)$ is the usual Heaviside step function. That is, all PBHs within a radius, $r(b, \theta, \Delta t)$, are assumed to produce exactly one burst of size $b$. In this work we set $P\left(b, N_{D}\right)=\mathrm{e}^{-N_{D}} N_{D}^{b} / b$ !, since a Poisson distribution should give a more realistic 
description of the chances of detecting a PBH burst of a given size. A similar approach is used in [16]. Substituting this back into equation (3), we now have for the expected number of PBH signal counts,

$$
n_{s}(b, \Delta t)=\frac{\rho_{\mathrm{pbh}} \tau}{8 \sqrt{\pi}} \frac{\Gamma\left(b-\frac{3}{2}\right)}{b !} I^{3 / 2} \int_{-1}^{1} \mathrm{~d} \cos \theta a(\theta)^{3 / 2} .
$$

Upper limits on the PBH density are set by using a standard maximum likelihood analysis to convert the number of detected and background bursts into an upper limit on $n_{s}$ for each burst size $b \geq 2$. Equation (4) can then be used to convert these limits into an upper limit on the PBH density.

\subsection{Theoretical model for the final stages of PBH evaporation}

The only piece still missing from equation (4) is a theoretical model for the PBH gammaray flux to be used for the factor $I$. The gamma-ray flux used in this work (from both direct emission and fragmentation products of quarks and gluons) is

$$
\begin{aligned}
& \frac{\mathrm{d} N^{2}}{\mathrm{~d} E \mathrm{~d} t}=6.24 \times 10^{23} \mathrm{GeV}^{-1} \mathrm{~s}^{-1}\left[\frac{1}{8}\left(\frac{Q}{E}\right)^{3 / 2}-\frac{3}{4} \sqrt{\frac{Q}{E}}-\frac{3}{8} \sqrt{\frac{E}{Q}}+1\right], \quad \text { for } E<Q, \\
& \frac{\mathrm{d} N^{2}}{\mathrm{~d} E \mathrm{~d} t}=10^{21} \mathrm{GeV}^{-1} \mathrm{~s}^{-1}\left(\frac{Q}{E}\right)^{4}, \quad \text { for } E \geq Q,
\end{aligned}
$$

where $Q$ is the energy of the peak quark flux over the last $\Delta t$ seconds of the PBH's life, given by $Q \approx 4 \times 10^{4}(1 \mathrm{~s} / \Delta t)^{1 / 3} \mathrm{GeV}[2]$. The above equations only assume the existence of known Standard Model particles (e.g. neither Higgs particles nor supersymmetric partners are included) and are only applicable in the final stages of $\mathrm{PBH}$ evaporation once its temperature has surpassed the rest mass of the top quark ( $\approx 26 \mathrm{~h}$ before total evaporation). If the assumptions about the final stages of evaporation are changed, the expected signal changes as well, altering the optimal energy and timescales to be searched. For example, a phase transition at some energy scale $\lambda$ could result in an exponential increase in the number of particle degrees of freedom for a limited energy range above $\lambda[2]$. In such a scenario the number of bursts at that energy scale would increase dramatically.

We can estimate the distance out to which the search in this work is sensitive by setting $N_{D}$ in equation (2) to one and solving for $r$. Doing this, we find $r \approx 0.6 \mathrm{pc}$, thus justifying the neglect of cosmological redshifting and absorption of gamma rays by extragalactic background light in the above calculations.

\section{Results}

A search has been made for bursts of high energy gamma-ray events with lengths of 1 , 3 , and $5 \mathrm{~s}$. The number of observed bursts of various sizes, $n_{m}(b)$, has been measured and the corresponding expected number of background bursts, $n_{b}(b)$ has been estimated as discussed in section 3.2. Bursts containing up to seven gamma-ray events have been observed. However, there is no significant excess above the expected background from statistical fluctuations of the arrival times of uncorrelated gamma-ray-like events (figure 2). Using a standard likelihood analysis, this null result is used to place an upper limit on the 


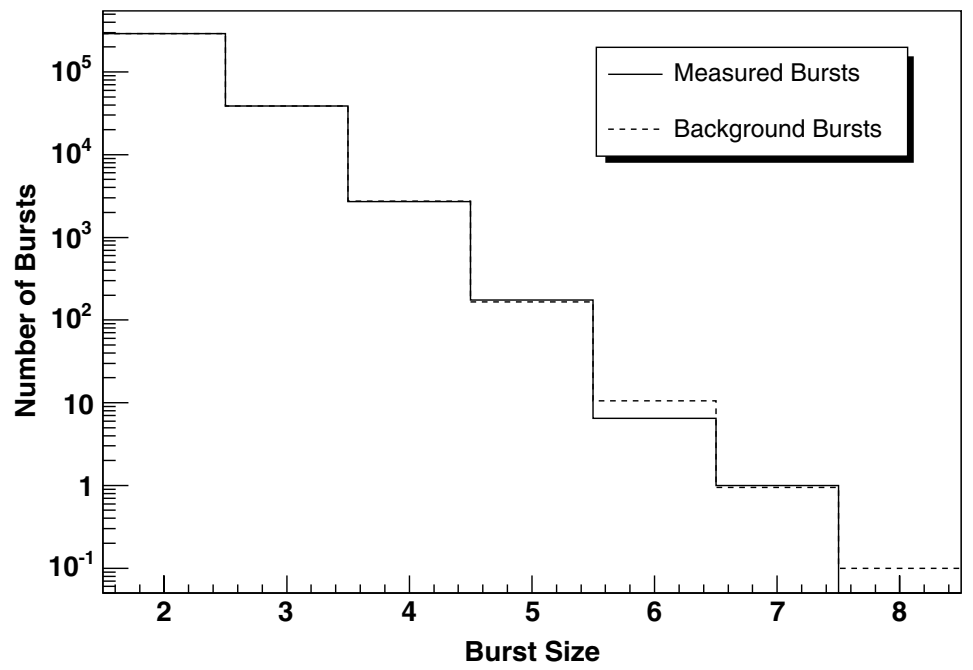

Figure 2. Number of bursts versus burst size for both the measured and background data. These data are for the most recent configuration of the 379PMT camera with $\mathrm{ZA}<20^{\circ}$ and a time window of $5 \mathrm{~s}$.

Table 1. The $95 \%$ and $99 \%$ upper limits on $\rho_{\mathrm{pbh}}$ in units of $10^{6} \mathrm{pc}^{-3} \mathrm{yr}^{-1}$.

\begin{tabular}{lll}
\hline$\Delta t(\mathrm{~s})$ & $95 \%$ upper limits & $99 \%$ upper limits \\
\hline 1 & 1.00 & 1.72 \\
3 & 0.94 & 1.59 \\
5 & 0.63 & 1.08 \\
\hline
\end{tabular}

number of $\mathrm{PBH}$ evaporations per unit volume per unit time in our local galactic region (table 1). The systematic uncertainty in the limits is estimated by adjusting the gain of the telescope in the simulations by $20 \%$. This leads to a systematic uncertainty of about $30 \%$.

\section{Conclusions}

We have made a direct search for the bursts of gamma rays expected from the evaporation events of PBHs. The null result enables us to place a limit on the $\mathrm{PBH}$ evaporation rate per unit volume, which extends to burst timescales of $5 \mathrm{~s}$ for the first time. A conservative likelihood analysis of the burst data in comparison with simulations leads to a limit on $\rho_{\mathrm{pbh}}$ of $1.08 \times 10^{6} \mathrm{pc}^{-3} \mathrm{yr}^{-1}(99 \% \mathrm{CL})$. Figure 3 places this result within the context of other searches for $\mathrm{TeV}$ and higher energy bursts from $\mathrm{PBH}$ evaporation. The limit set in this work is more than a factor of two below the limit set in a previous search through Whipple data [15]. However, this previous limit was set using Gaussian statistics, so no use could be made of the fact that no bursts containing more than four events were seen. Applying Poisson statistics to the data in reference [15] yields a more stringent limit of $1.84 \times 10^{6} \mathrm{pc}^{-3} \mathrm{yr}^{-1}$, which is comparable to the $\Delta t=1 \mathrm{~s}$ limit in this current work. This present work searched around five and a half years of gamma-ray data for bursts; the previous Whipple result [15] was a search extending just over five years. Combining 


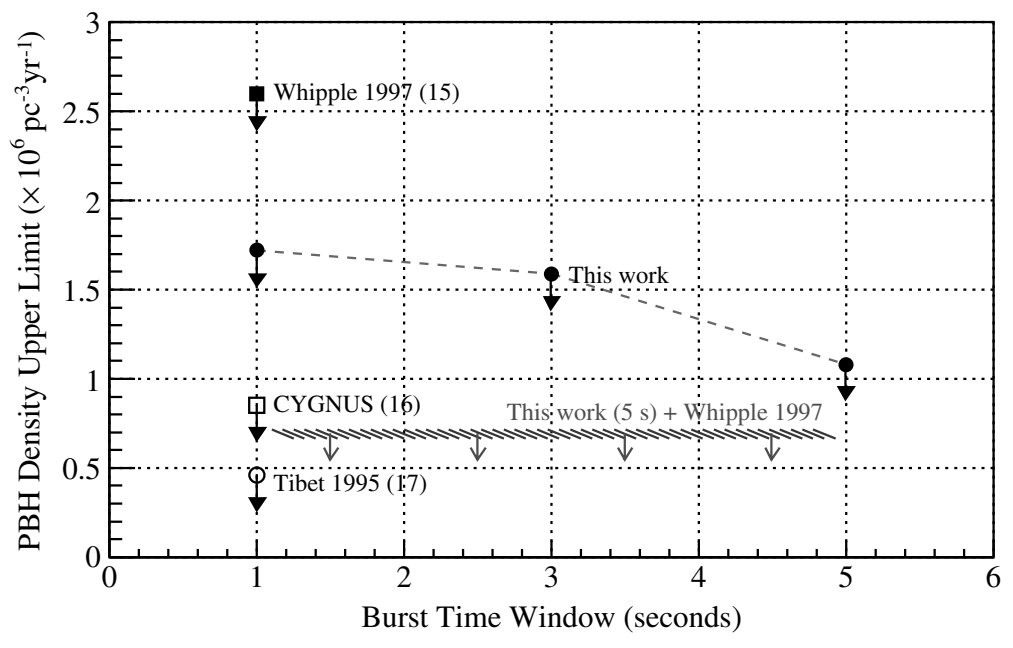

Figure 3. Upper limits on PBH density from direct burst-search experiments.

the results from this search across a range of burst lengths extending to $5 \mathrm{~s}$ with the previous Whipple search yields an upper limit of $0.69 \times 10^{6} \mathrm{pc}^{-3} \mathrm{yr}^{-1}$. This new ACT limit is comparable to those set by air-shower measurements using the Tibet array at $\sim 10 \mathrm{TeV}[17]$ and CYGNUS at $\sim 50 \mathrm{TeV}$ [16]. However, the new ACT limit explores both a lower energy range $(\sim 1 \mathrm{TeV})$ and a wider range of timescales, providing additional constraints on models of PBH evaporation.

Restrictive limits on the density of PBHs come from observations of the overall background radiation that they leave in the universe from their evaporations. As discussed in a recent review of potential dark matter candidates by Overduin and Wesson [18], limits can be set from the observed gamma-ray background, which essentially integrates together all the radiation from evaporating PBHs over the history of the universe [19]. The resulting limits provide an average limiting density for PBHs. This isotropic value requires a clustering model for direct comparison with our measurements in the local galaxy. The gamma-ray background limits have been estimated to correspond to a local rate of $\sim 10 \mathrm{pc}^{-3} \mathrm{yr}^{-1}$ for certain models of clustering [2]. However, recent work suggests that PBH clustering could be as many as 15 orders of magnitude greater than previously thought [20]. With such strong clustering the direct limit of this work would be about 10 orders of magnitude below the indirect limits set from the diffuse gamma-ray background. There are also significant limits on $\mathrm{PBH}$ density claimed on the basis of the absence of antiprotons below the kinematic production threshold in interstellar collisions [21]. Again there is a significant model dependence in the values of these limits derived from indirect observations.

In this work we have focused on a direct search for PBH decay since the effects of clustering might produce some evaporation signals in our local neighbourhood even though the isotropic density seems quite low from measurements of background radiations. To quote the original statement of Page and Hawking made 30 years ago [22], 'the best prospect for detecting a primordial black hole seems to be to look for the burst of hard gamma rays that would be expected in the final stages of the evaporation of the black hole'. This remains an exciting goal for ground-based gamma-ray telescopes. 


\section{Acknowledgments}

We are grateful for the technical assistance of E Roache and J Melnick. The VERITAS collaboration is supported by the US Department of Energy, the National Science Foundation (NSF), the Smithsonian Institution, NSERC (Canada), PPARC (UK), and the Science Foundation of Ireland.

\section{References}

[1] Hawking S W, 1974 Nature 24830 [SPIRES]

[2] Halzen F et al, 1991 Nature 353807 [SPIRES]

[3] Carr B, 2003 Quantum Gravity:From Theory to Experimental Search ed D Giulini, C Kiefer and C Lammerzahl [astro-ph/0310838v1]

[4] Maki K, Mitsui T and Orito S, 1996 Phys. Rev. Lett. 763474 [SPIRES]

[5] Porter N A and Weekes T C, 1979 Nature 277199 [SPIRES]

[6] Hagedorn R, 1968 Nuovo Cim. LVIX 1027 [SPIRES]

[7] LeBohec S et al, 2005 Astropart. Phys. 23235 [SPIRES] [astro-ph/0501199v1]

[8] Heckler A, 1997 Phys. Rev. D 55480 [SPIRES]

[9] Cawley M and Weekes T C, 1995 Exp. Astron. 67

[10] Bradbury S et al, 1999 Proc. 26th Int. Cosmic-Ray Conf. (Salt Lake City) vol 5

[11] Hillas A, 1985 Proc. 19th Int. Cosmic-Ray Conf. (La Jolla)

[12] Reynolds P, 1993 Astrophys. J. 404206 [SPIRES]

[13] Lessard R et al, 2001 Astropart. Phys. 151 [SPIRES]

[14] Kertzman M and Sembroski G, 1994 Nucl. Instrum. Meth. A 343629

[15] Connaughton V et al, 1998 Astropart. Phys. 8179 [SPIRES]

[16] Alexandreas D et al, 1993 Phys. Rev. Lett. 712524 [SPIRES]

[17] Amenomori M et al, 1995 Proc. 24th Int. Cosmic-Ray Conf. (Rome) vol 2

[18] Overduin J and Wesson P, 2004 Phys. Rep. 402267 [SPIRES]

[19] Carr B and MacGibbon J, 1998 Phys. Rep. 307141 [SPIRES]

[20] Chisholm J, 2005 Preprint astro-ph/0509141v1

[21] Barrau A et al, 2003 Phys. Lett. B 551218 [SPIRES]

[22] Page D and Hawking S W, 1976 Astrophys. J. 2061 [SPIRES] 